\title{
Home telehealth in older patients with heart failure - costs, adherence, and outcomes
}

This article was published in the following Dove Press journal:

Smart Homecare Technology and TeleHealth

18 September 2014

Number of times this article has been viewed

\section{Susanna Spinsante \\ Dipartimento di Ingegneria dell'Informazione, Università Politecnica delle Marche, Ancona, Italy}

Correspondence: Susanna Spinsante

Dipartimento di Ingegneria dell'Informazione (DII), Università Politecnica delle Marche, Via Brecce Bianche 12, 60131, Ancona, Italy Tel +39 07l 2204894 Fax +39 07I 2204224 Email s.spinsante@univpm.it

\begin{abstract}
This short review discusses the role of telehealth technologies in the management of older patients with heart failure, from different perspectives. Instead of providing a systematic overview of existing literature in the field, this paper provides evidence for a simple, but effective, paradigm upon which a telehealth system may be built, and highlights how such a model may successfully apply to heart failure management, to improve patients' quality of life after discharge, increase independency, and reduce readmissions and costs for the public health institutions. A few examples are discussed, to show the real applicability of the proposed model and further confirm the effectiveness of telehealth, when properly designed and tailored to users' needs.
\end{abstract}

Keywords: remote health care, workflow, requirements

\section{Introduction}

Traditional health care paradigms of most developed countries are experiencing dramatic changes in modern times, showing a rapid shift from in-hospital care to more advanced home health care solutions, according to new, decentralized models. These changes are driven by several and differentiated factors, either economical, social, or technological ones. In the economic perspective, the still ongoing global crisis is putting big pressure on the welfare and health care-related national systems. The strong reduction of the available financial resources collides with a growing demand for health care services and facilities, due to an increased incidence of elderly population and changed lifestyles, leading to a longer life duration but also a stronger impact of chronic diseases. In the social perspective, most of the developed countries are experiencing a demographic shift; as an example, the life expectancy for males and females in Europe has increased from 45.7 and 49.6 to 75.0 and 79.9 years, respectively, in less than a century. ${ }^{1}$ Looking at long-term projections, the incidence of those over the age of 85 years among the older adults is set to increase at an even faster pace; moreover, there is a growing concern about the low birth rate in industrialized countries. Demographic changes affect a wide range of economic and social fields, as well as policies concerned with health, social welfare, housing, and many other issues. Further, new challenges and trends regarding health care services are emerging in many developed countries, such as: the demand for increased availability of care services outside hospitals and medical institutions, and into patients' own premises; the need for improved efficiency of the services, to maintain acceptable quality despite reduced financial resources; and the difficulties and costs in recruiting personnel specialized in elderly care, especially to deliver home assistance. Moreover, there is a global trend of individuals being more 
and more interested in self-managing their health and willing to age at home, rather than in an institution. ${ }^{2}$ At the same time, most of the available research reports ${ }^{3}$ and studies ${ }^{4-6}$ claim that, despite a necessary significant financial investment at a first stage, the adoption of information and communication technologies (ICT), in the delivery of remote health services to populations, can really enable strong savings while preserving quality and efficacy of services and treatments. In the broader field of telemedicine and/or telehealth, several solutions and systems have been conceived and implemented in various forms over many years; telehomecare or home telehealth ${ }^{7}$ is often merged with different approaches, such as those related to Ambient Assisted Living.

Telehealth (also known as remote health) in general refers to a number of technologies, systems, and applications that may be adopted to provide remote support of health care at home. Such support requires a set of basic functionalities, such as remote consultation and diagnosis (usually implemented through audio-video communication facilities), as well as the possibility of collecting data for the monitoring of health parameters and vital signs (eg, blood pressure, heart rate, and seizure risk).

According to the 2011 Telehealth Advancement Act, Section 2290.5 of the California Business and Professions Code, telehealth is:

the mode of delivering health care services and public health via information and communication technologies

[...] while the patient is at the originating site and the

health care provider is at a distant site [...] and includes

synchronous interactions and asynchronous store and

forward transfers. ${ }^{8}$

Patient's related data are usually gathered by means of biomedical sensors and devices located at the remote patient's home and are transferred to a medical operator, or a nurse, through a collecting centralized node over a wireless or wired data connection. Telehealth may be applied to a number of different areas requiring health assistance, such as chronic diseases, heart-related diseases, management of diabetes, nursing, dermatology, or ophthalmic problems, up to telerheumatology, teleneurology, and telepsychiatry. ${ }^{9-14}$

This paper focuses on home telehealth technologies applied to monitor older adults suffering from heart failure (HF). HF represents a major burden on many health care systems, especially in developed countries. As the number of patients affected by HF increases, the cost of hospitalization alone impacts significantly on the overall cost of this disease. Readmission rate, and hospital length of stay, are assumed as quality markers of HF care, that are to be optimized through suitable initiatives, among which telehealth may play a crucial role. ${ }^{15}$ The disease process of HF inherently requires demanding and close attention to vital parameters, diet, and medication compliance, to prevent acute decompensation episodes. As a consequence, remote patient monitoring (RPM) may represent a key strategy for HF disease management, to optimize both the quality of care and the outcomes obtainable. The target of this review is to discuss telehealth approaches and technologies for HF disease management, in the perspectives of cost, adherence, and outcomes. In this paper, a concise overview of significant approaches to telehealth is first provided, by presenting some solutions and technologies focused on HF management. Then, adherence and outcomes of telehealth solutions for managing older patients suffering from HF are discussed, and a cost-related evaluation of telehealth applied to HF proposed. Finally, the main conclusions of the work are drawn.

\section{Telehealth solutions for HF in older adults: some experiences}

In most of the developed countries, $\mathrm{HF}$ alone contributes to the majority of the health care expenditure, basically due to inpatient care. Episodes of acute decompensated HF are the most common reason for hospital admission. Ideally, these should be predicted, to allow early intervention and avoid hospitalization. Moreover, as acute decompensation is strongly associated with future mortality, effective strategies to avoid such occurrences could potentially translate into improved survival ${ }^{16}$ Getting good outcomes in $\mathrm{HF}$ treatment requires patients' cooperation, in being extremely compliant and vigilant and concurring with the goals of therapies and care. In fact, studies show that, typically, because of dietary and medication noncompliance, a significant proportion of patients suffering from chronic HF (CHF) experience an episode of acute decompensation which may lead to congestive symptoms and possible rehospitalization. ${ }^{17,18}$

\section{Remote health monitoring workflow and components}

Regardless of the specific technologies and system architecture used, a typical workflow of remote health monitoring includes five basic steps, as shown in Figure 1. These comprise data collection, data transmission, evaluation, notification, and intervention. They are common to almost any telehealth system, possibly with a focus on a specific step, according to the particular nature of the system itself and the target users addressed by the services supported 


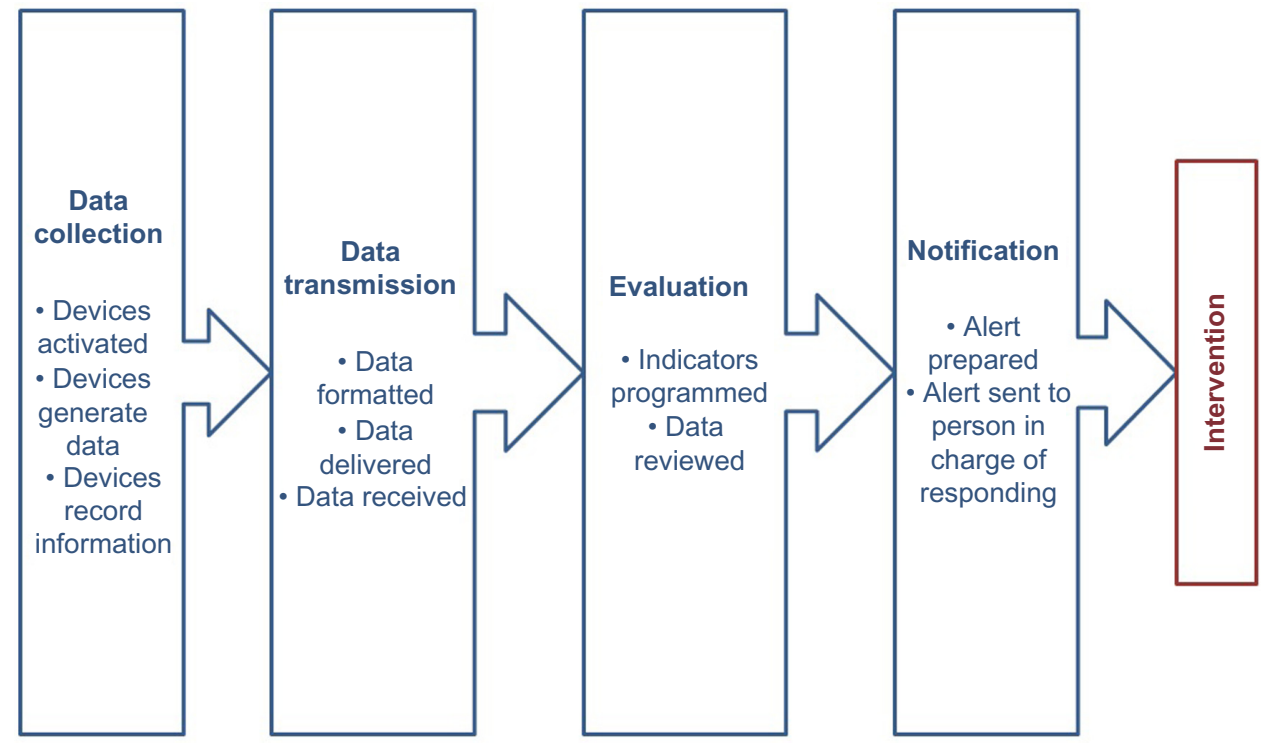

Figure I Reference workflow of remote health monitoring.

(eg, if patient-centric, or oriented to support caregivers and medical operators).

Each step in the workflow may include different sub-steps, the number and complexity of which may change according to the general level of complexity and specificity of the whole system to be implemented. Data are collected through different devices (biomedical monitors, wearable sensors, and even ambient sensors) and may include vital signs, blood glucose levels, responses to specific health condition questions or general health questions, as well as patient location. The information gathered is then formatted, delivered, and received by providers, family caregivers, and third parties via different modes of communication and different platforms, such as those based on cloud technologies. Algorithms and/or health care workers review the data to determine if any alarm condition holds or to check if any of the parameters observed show a negative trend that may be reason for concern. If needed, family caregivers, clinicians, third parties, or the patients themselves are notified of a potential problem, through either the device itself or an intermediary health care worker. They can then take action, if an intervention is needed. Such a workflow represents a common paradigm for telehealth solutions; different combinations of technologies and algorithms may be chosen to implement it. ${ }^{19}$

Providing an exhaustive overview and comparison of different telehealth experiences is a tricky task: over 40 years of research has yielded a wealth of data about the costeffectiveness and efficacy of many telehealth applications. Over 1,000 evaluative studies have been published in leading journals devoted to telemedicine. As a general remark, for any possible telehealth service and architecture to be designed, a large literature of similar or comparable experiences exists and can provide useful insights about possible advantages and disadvantages and provisional and forecast data about expected outcomes and impact on patients. Many comprehensive overviews of possible monitoring approaches for $\mathrm{HF}$, from structured telephone monitoring to monitoring with home-based portable technology or specially designed wearable and implantable devices, are available in the literature, such as in the study by Bhimaraj, ${ }^{6}$ or about the specific components of the homecare intervention, such as in the study by Jaarsma et al. ${ }^{20}$

Suitably designed (ie, compliant, user-friendly, safe) devices for RPM have been shown to increase the patients' role in the management of their own health, improve chronic disease management, and reduce the incidence of acute episodes. By using a variety of integrated or standalone RPM devices, up-to-date information on patients' chronic disease and/or post-acute care status (including vital signs, heart rate, blood glucose levels, medication management, mental health, physical and cognitive fitness), and other data (such as patient's location, or ambient parameters) can be transmitted to family caregivers, providers, or third parties in charge of patient monitoring. Clinicians, or properly trained individuals, can then intervene with coaching actions or by adjusting the course of treatment.

There exist different types of integrated devices for RPM that act as aggregators of data originated from multiple peripheral sensors (eg, blood pressure cuff, weight-monitoring scales, glucose monitor, pulse oximeter, 
prothrombin time/international normalized ratio meter, thermometer, electrocardiogram, peak flow meter, stethoscope, pedometer) that are wirelessly connected or plugged directly into the concentrator. Some instruments can also self-activate and alert patients and caregivers that a test or medication must be taken. Data are subsequently transferred to health care professionals, when they are categorized through patient-specific algorithms to evaluate risk and lead to appropriate actions. Many of these tools store previous outcomes and make them accessible through web-based programs, so that a time comparison of the collected data over a given interval may be performed in order to analyze the patient's health trend.

Some representative telehealth experiences designed to assist patients affected by HF at their own home premises are briefly reported here, and selected mainly because of their impact in terms of practical and widespread implementation as well as their research-oriented contributions.

\section{The Veterans Health Administration (VHA) study}

Many demonstration and pilot projects aimed at proving the effectiveness of home telehealth have been completed or are underway. One of the most comprehensive studies to date was performed by the VHA, incorporating 4 years of data on over 17,000 patients. ${ }^{21}$ The VHA's program is in charge of ensuring support to care for veterans needing assistance, especially those living in rural areas, who represent almost $32 \%$ of the veteran population. After a patient is enrolled into the program, his or her care coordinator selects the appropriate home telehealth technology, gives the required training to the patient and caregiver, reviews telehealth monitoring data, and provides active care or case management (including communication with the patient's physician). Typically, an individual care coordinator manages a panel of between 100 and 150 general medical patients, or 90 patients with mental health-related conditions. Once the patient's underlying chronic condition has been assessed, the care coordinator selects the appropriate vital signs, objective parameters (such as blood glucose), and other disease-related information to acquire from the home for ongoing monitoring and management purposes. The care coordinator then decides which technology is best suited to collect these data. The program uses remote monitoring devices in veterans' homes to communicate health status and to capture and transmit biometric data that are monitored remotely by care coordinators.

The program enrolls veterans with diabetes, congestive HF, hypertension, posttraumatic stress disorder, and other conditions. The VHA Office of Telehealth Services uses health informatics, disease management, and telehealth technologies to facilitate access to care and improve health outcomes in three main ways:

- clinical video telehealth: interactive video technologies are applied, for the real-time delivery of physician visits to distant clinics, to make diagnoses, manage care, perform check-ups, and provide care in polytrauma, mental health, rehabilitation, and surgical consultations;

- store-and-forward telehealth: to support the acquisition, transmission, and storage of prerecorded information (sound, data, images), such as X-rays, video clips, and photos, between providers and specialists in radiology, dermatology, and retinopathy (to enable a second-levelopinion option); and

- care coordination/home telehealth: biomedical monitoring devices and sensors are used to capture the patient's physiological data related to symptoms, and vital signs, in the home environment. Data are then transmitted to health care providers for review and appropriate coordination of care. ${ }^{22}$

The most commonly used technologies are messaging and monitoring devices ( $85 \%$ ), followed by video-telemonitors (11\%) and videophones (4\%). Messaging devices ask patients questions to help assess their health status and capabilities for disease self-management. Monitoring devices record vital signs data, whereas videophones and video-telemonitors facilitate audio-video consultations at home.

In the years from 2004 to 2007, up to 4,089 patients suffering from congestive HF were treated by the VHA telehealth program: the reduction in health care resource utilization (defined as hospital days of stay) amounted to $25.9 \%{ }^{23}$

The VHA has found that an enterprise-wide home telehealth system is an appropriate and cost-effective means of managing chronic care patients in both urban and rural settings. Besides the reduction in bed days of care, studies that compared data from the year before entering the program and 6 months post-enrollment show a $20 \%$ reduction in number of admissions and a mean satisfaction score rating of $86 \%$. Decreases in health resource utilization were largest in highly rural (50.1\%) and urban (29.2\%) areas for mental healthrelated conditions and for patients with multiple conditions. Patients' acceptance of the remote monitoring service was high, with only $10 \%$ declining services. ${ }^{21,24}$

The intent of the VHA is to make its telehealth program available in the course of time to an ever larger population; this implies scaling a solution that reproduces the positive and promising financial and care outcomes from pilots, while 
ensuring sustainability of the clinical, technological, and business processes necessary to support home telehealth.

\section{The CAtalan Remote Management Evaluation (CARME) Study}

It is well known from the research literature that telemonitoring may allow achievement of a decrease in HF-related hospitalizations, an improvement in quality of life and in evidence-based prescribing, and even a reduction in all-cause mortality. Different telemonitoring systems in patients with HF have shown a global tendency toward a reduction in hospitalizations, although there is a great level of heterogeneity in the results achieved by means of different technologies. ${ }^{25-28}$

Between 2007 and 2009, an RPM program for patients with HF already managed in a specific multidisciplinary $\mathrm{HF}$ unit was implemented in the Spanish Catalan region, to evaluate its effectiveness.

HF patients were monitored over 1 year, through a telehealth solution based on TV and a broadband Internet connection. Patients were randomized to two groups: one using the system as a disease-management tool (provision of educative videos, messages, charts, questionnaires), and the other group using the system together with devices for telemonitoring of blood pressure, pulse rate, and weight. Hospitalizations were compared during similar periods of time ( 1 full year) before and after the inclusion in the program. A total number of 92 patients were studied. Most patients were in moderate-to-severe disease stages (New York Heart Association [NYHA] class II [81.5\%] or III [18.5\%]).

The results of the project, named CARME, showed that the telemedicine program led to a significant reduction in the number of hospital admissions and days in the hospital for $\mathrm{HF}$ and other cardiac causes. For patients in the group with only disease telemanagement tools, all-cause admissions increased about $30 \%$, but admissions due to HF were reduced by $80 \%$. On the contrary, patients in the group with both management and telemonitoring tools experienced a significant reduction in both all-cause hospital admissions (63\%) and in HF admissions (75\%).

The program also resulted in a perception of better quality of life for the patients..$^{29}$ At the beginning of the study, more than one-half of the patients $(55.88 \%)$ said that their quality of life was "medium" or "low". After 6 months, this number decreased to $29.41 \%$, and to $22.06 \%$ at the end of the study. Furthermore, the percentage of patients that considered their quality of life to be "medium-to-high" rose from $43.61 \%$ at the beginning of the study to $77.94 \%$ after 1 year, and almost one-third (27.94\%) considered it as "almost excellent". Users expressed high satisfaction about the system and the available tools, especially the self-monitoring chart, the scale, and the sphygmomanometer provided for autonomous usage at home. Positive changes were observed in patients' behavior, especially for the control of blood pressure and weight. After the pilot study, $65 \%$ of the patients wished to continue with telemonitoring, showing confidence in the system, a high degree of satisfaction with the tools, and positive behavioral changes. From the point of view of the technologies involved, the project showed real feasibility of the solution and relative easiness of implementation and duplication.

\section{A randomized controlled trial in Bilbao (Basque Country, Spain): the TELBIL study}

The adoption of structured telephone support, or telemonitoring in the home, can improve the quality of the care for patients affected by HF moving from the management of destabilization events within hospitals to a model focused on the maintenance of the basal status. Telemonitoring in the framework of the TELBIL study (Evaluation of a primary care-based telemonitoring intervention for home care patients with heart failure and chronic lung disease: a randomised controlled trial) ${ }^{30}$ consisted of daily transmissions of several self-measured clinical parameters, collected by using a personal digital assistant with the help of the caregivers from the patients' premises. Additionally, patients completed a brief health status questionnaire aimed at assessing the perception of personal medical and functional condition, and also concerning compliance with medication and diet. All data were stored on a web-based platform accessible by health professionals. The system comprised personalized alerts, specifically set for each patient, when the recorded parameters fell outside the pre-established limits (which could be adjusted over time through the web platform). The main objective of the study was to evaluate the effect of a primary care-based telemonitoring intervention on the number and length of hospital admissions. ${ }^{30}$ The primary outcome measure was the number of hospital admissions that occurred at 12 months post-randomization. The impact of telemonitoring on the length of hospital stay, the use of other health care resources (emergency department attendances, home visits by primary care professionals, appointments at the health center or with specialists, and telephone calls), and mortality were also explored. Across 20 health centers, 58 in-home patients diagnosed with HF and/or chronic lung disease, aged 14 years or above and with two or more hospital admissions in the previous year, were recruited. The intervention group included 28 patients and the control group 30. Patient 
baseline characteristics were similar in both groups. Of the 21 intervention patients followed-up for 1 year, 12 had some admissions (57.1\%), compared to 19 of 22 controls $(86.4 \%)$. The mean hospital stay was overall 9 days among controls, and for cause-specific admissions 9 versus 11.2 days, both without statistical significance. There were more telephone contacts in the intervention group than in the control group, but fewer home nursing visits, though the difference was not statistically significant.

\section{Experiences with non-positive outcomes}

Trials and experiences like the ones discussed above show that RPM may help to detect early signs of cardiac decompensation, allowing optimization of and adherence to treatments in CHF. Disease-management programs, mostly provided via HF clinics, have been reported to provide better care to $\mathrm{CHF}$ patients and to reduce health care utilization. However, it is necessary to say that not all the trials and experiences provided positive outcomes and results. As an example, Koehler et al $^{31,32}$ presented a study in which it was shown that in ambulatory patients with $\mathrm{CHF}$, remote telemedical management compared with usual care was not associated with a reduction in all-cause mortality.

Seven hundred and ten stable CHF patients in NYHA functional class II or III with a left ventricular ejection fraction $\leq 35 \%$ and a history of HF decompensation within the previous 2 years, or with a left ventricular ejection fraction $\leq 25 \%$, were enrolled and randomly assigned to remote telemedical management or usual care. ${ }^{31}$ Remote telemedical management involved the use of portable devices for electrocardiogram, blood pressure, and body weight measurements connected to a personal digital assistant that sent automated encrypted transmissions via cell phones to the remote centers. Data were transferred daily to physician-led telemedical centers that were active on a 24 hours per day, 7 days per week basis.

The primary endpoint was death from any cause. The first secondary endpoint was a composite of cardiovascular death and hospitalization for HF. Compared with the usual-care group, over a median follow-up of 26 months, there was no reduction in the risk of hospital readmission (for any reason) or all-cause mortality in HF patients assigned to telemonitoring who had been recently hospitalized for worsening HF. In the study by Takahashi et al, ${ }^{33} 205$ participants with a mean age of 80.3 years were enrolled. Subjects were randomized to telemonitoring with daily input versus patient-driven usual care. Telemonitoring was accomplished by daily biometrics, symptom reporting, and videoconference. The system that was used had real-time videoconference capability and peripheral devices (weight-monitoring scales, blood pressure cuff, glucometer, pulse oximeter, and peak flow). Patients performed daily 5- to 10-minute monitoring sessions for symptoms and biometric information, then the system worked asynchronously to transmit data to a web health site, which was then reviewed by the health care team daily, including weekends and holidays.

The primary outcome of hospitalizations did not differ between the telemonitoring group (63.7\%) and the usual-care group (57.3\%). No differences were observed in secondary endpoints, including hospitalizations, visits, and total hospital days. Mortality was even higher in the telemonitoring group $(14.7 \%)$ than in the usual-care group $(3.9 \%)$, for reasons that are unknown.

It is neither easy nor appropriate to try to find a single motivation for experiences that did not provide positive outcomes. In any case, at least from the point of view of the technologies adopted in home telemonitoring, the studies discussed, ${ }^{31-33}$ and many others that can be found in the literature, show that approaches that use technology to help and improve the work of health assistants (such as nurses) provide more favorable outcomes than those trying to make the patient completely autonomous in the management of health diseases. Further, it is possible to state that, even when no difference in the time to rehospitalization or emergency visit between those who received telemonitoring versus usual care emerges, telemonitoring may act as an important adjunct to home health care services to improve health status. ${ }^{34}$

\section{Adherence and outcomes of telehealth solutions for HF}

It is widely recognized that medication nonadherence contributes, in the range of $33 \%-69 \%$, to medication-related hospital admissions, and to about $23 \%$ of all the nursing home admissions. Medication adherence, especially in the case of HF episodes, falls within the domain of post-acute care management, indicating that care management, such as that required after a hospital stay, is in need of improvement. Many recently hospitalized older adults do not recover as expected, because of the lack of continuous and guided monitoring when back at home due to a number of unavoidable home- and self-care challenges that too often result in hospital readmissions within weeks after discharge. In its turn, this leads to an excessive utilization of health care and reductions in patient independence.

Due to the significant margin for improvement, hospital readmissions rates are currently a key focus area for health 
systems and health policy initiatives. A quite significant amount (almost 20\%) of all hospital admissions are actually readmissions, and a majority of these could be avoided through a more effective management of health care after discharge, thus providing estimated savings of several billion dollars. ${ }^{35}$ Factors that contribute to excessive hospital readmissions include service fragmentation and poor communication among and between health care settings and care providers and poorly delivered and/or understood discharge instructions and follow-up. By improving coordination across the continuum of care and promoting seamless transitions from the hospital to home, skilled nursing care, or home health care, avoidable readmissions rates can be decreased. Personal health records, also known as electronic health records, and RPM technologies can effectively support or enable suitable care models. Similar to applications in chronic disease management, RPM technologies enable better post-acute care self-management, early intervention, and other improvements mentioned in the previous section. This can be accomplished in a variety of ways, as described in Table 1, compiled from analysis of market trends and solutions. ${ }^{36}$

Several experiences in home monitoring of elderly patients with congestive $\mathrm{HF}^{37,38}$ provided positive outcomes, in terms of reduction of the rehospitalization rate and improvements in the regular consumption of drugs and medications by the patients. It has been shown that up to $64 \%$ of HF hospitalizations could be prevented by adherence to the prescribed medication and diet, but up to $90 \%$ of HF patients do not fully adhere to their recommended regimens. ${ }^{39}$

Table I Improvements of health self-management enabled by remote patient monitoring capabilities

\begin{tabular}{ll}
\hline Remote patient monitoring & Enabled improvements \\
capability & - Disease knowledge \\
\hline Therapy management & - Vital signs and self-reporting \\
& - Management of medication \\
Health and preventive behavior & - Nutrition \\
management & - Physical exercise \\
& - Cognitive exercise \\
& - Social interaction \\
Daily life management & - Stress reduction \\
& - Prolong living at home \\
& - Seal with disease-related implications \\
& - Support in daily life by friends, family \\
Acute event management & - Be prepared for acute events \\
& - Call for help \\
\hline
\end{tabular}

Patients have highly varied needs for remote monitoring technologies. Some patients want a simple, inexpensive, portable technology, while others may require an expensive, integrated, home-based technology. There is a wide spectrum of technologies in between, from simple to complex, that meet the needs of nearly all patient segments in the most appropriate way. The ability to augment patient self-management tools and skills is critical to the value of integrated RPM devices. As older adults progress through the natural aging process, they may experience some degenerative effects, including reduced vision, varying degrees of hearing loss, and psychomotor impairments, as well as decreasing attention, memory, and learning abilities. These phenomena affect the way older users may interact with personal computers, mobile devices, and technological equipment in general. It is generally recognized that advanced health care solutions based on innovative ICT should be properly designed and exploited, in order to implement preventive health care initiatives (to limit the impact of chronic diseases), to cut costs, and to introduce new services and techniques for early diagnosis, targeted therapy, and remotely assisted home care. Despite this understanding, however, most of the solutions tested and provided up to now present some basic limitations, which are mainly due to the usually complex architecture they require, and to the fact that patients are typically not familiar or comfortable with the use of technology equipments, or do not trust them enough. ${ }^{40,41}$ Relying on well-known and comfortable technologies, such as the TV set, ${ }^{42}$ enables the older user's expected requirements to be met: the patient's physiologic data are collected without the need for any further action besides data measuring (ie, switching on the medical device, measuring, switching off the device), according to a blind approach that minimizes the user's interaction with the system. Such a capability is achieved by exploiting the Open Services Gateway initiative (OSGi) architecture and its Declarative Services paradigm, running on an embedded platform that also provides support for Bluetooth connectivity, and exploiting the TV set for the consultation of data at home.

It is important to highlight that the selection of the proper technology to implement a telehealth system may have a strong impact on its successful adoption. In many countries, even the most industrialized ones, older adults usually live in rural or suburban areas, within small communities, where health care facilities may be not easily accessible, especially those specialized centers involved in the management of specific diseases such as HF. In such a scenario, mobile networks have already surpassed the personal computer as a means of extending health care capacity in 
underserved communities. Mobile devices may be equipped with health-related sensors, and provide visibility into what patients are actually doing - not just what they say they are doing. Further, their natively enabled connectivity makes it possible to track patient health via wireless medical devices and support immediate and live patient-health provider communication in order to deliver more effective care.

Some studies tried to evaluate patient satisfaction with the use of telemedicine to access care, and with the use of telecommunications technologies to connect with specialists and other health care providers. ${ }^{43,44}$ Usually, the degree of satisfaction expressed by patients who are actively involved in RPM experiences is quite high: it may vary slightly with the specific service accessed through telemedicine, but, overall, patients have responded well to its use. The source of satisfaction for most patients is the ability to see a specialist trained in the area most closely related to the patient's condition, the feeling of getting personalized care from a provider who has the patient's interest in mind, and the ability to communicate with the provider in a very personal and intimate manner over the telecommunications technologies. ${ }^{45}$ Systematic reviews addressing patient satisfaction, in relation to patient age, sex, race, income, education, and insurance, found an overall satisfaction rate of up to $98.3 \%$. A randomized trial of telenursing to reduce hospitalization for $\mathrm{HF}^{46}$ demonstrated that patient self-care adherence, medications, health status, and satisfaction do not significantly differ between groups (patients provided with RPM services and inpatients), meaning that the mediation of technology in the contact between the patient and the nurse does not have a negative impact on the patient's behavior toward the management of health diseases. As discussed in the review by Gorst et $\mathrm{al},{ }^{47}$ the common reasons for withdrawal from trials and studies, by patients offered telehealth only, are related to the unwillingness to use telehealth devices, health deteriorations, and technical problems. Technical problems, believing telehealth to be unnecessary, preference for in-person care, technology anxiety, difficulty remembering to interact with the system, need for technical support, and finding telehealth to be a repetitive process are among the individual barriers to telehealth most commonly reported by patients. It is evident that the design of the technological equipment, devices, and systems selected to provide a telehealth service is critical to the effective and positive adoption by the users. Furthermore, uptake and withdrawal of telehealth experiences may be impacted by the health status of the individual: patients who are very unwell and those who are relatively well may not want to use telehealth because they think either that they are too ill to use it or not ill enough. A careful and detailed evaluation of the user's specific needs and conditions is another basic element for the success of any telehealth service provisioning. As a matter of fact, health care professionals have a key role to play in patient recruitment to telehealth interventions, by screening patients for whom they consider telehealth would be beneficial. At the same time, some health care providers remain to be convinced about telehealth. For example, the study by Joseph et $\mathrm{al}^{48}$ showed that some nurses were concerned that telehealth would make them redundant, thus they were less enthusiastic than patients in the initial phases of telehealth implementation.

\section{Cost-related evaluation of telehealth applied to HF}

The impact of ICT on the health care sector is a double-faced phenomenon. On one hand, many effective and significant advances are continuously taking place, especially in the field of medical treatments and devices designed for their delivery. Also, the management of health data and patients' records is experiencing a kind of digital revolution, thanks to the widespread introduction of the electronic health record to gradually replace traditionally heterogeneous, and often partially hand-processed, data management services. On the other hand, although appropriate technology is available, very limited spreading of remote health monitoring solutions is evidenced, especially among those users that could really benefit from it, such as elderly people or physically disabled people..$^{40,49,50}$ In terms of cost savings, a study by economist Robert Litan, in 2010, reported that a more widespread use of remote technology over the following 25 years, including home telehealth, could save as much as US \$197 billion, by helping to manage care coordination for patients with chronic diseases, such as CHF and diabetes. ${ }^{51}$ The United Nations reported in 2012 that the number of people older than 60 years was expected to reach $22 \%$ of the world's population by $2050 .{ }^{52} \mathrm{HF}$ is a typical disease of the older adult population and, according to the global trend of longevity increase and aging of societies, especially in developed countries, the prevalence of HF acute events is expected to significantly increase the total annual costs related to HF management. The same concerns are shared by several European countries, where the trend of readmission rates is also monitored. To limit the cost burden of avoidable admissions, many countries are supporting and subsidizing the large-scale deployment of telehealth delivery services. ${ }^{53}$ In an effort to reduce 30 -day HF readmissions, and improve the overall progress in patient care, the use of 
RPM technologies for a successful transition from hospital to home is valued as a key success factor.

Technology may help in limiting the impact of costs faced to provide social and medical services: some assistance requests may be solved, for example, without moving health operators, by establishing a direct and live, though remote, communication session between doctors and patients. With monitoring and data transmission both occurring daily, patients might be able to avoid numerous trips to a physician's surgery, and physicians could quickly act and tailor medical treatments to variations of the patient's health condition. Despite the well-recognized positive effects that a widespread adoption of telemedicine could bring, not so many legacy systems are yet operating. As stated in the previous section, several pilot and experimental initiatives have been carried out, but it is possible to say that a standard and systematic adoption of telemedicine, as a way of delivering health services, is still lacking, both in the USA and in European countries.

There are several factors that could contribute to the translation of telehealth pilots or experimental trials into daily life practice: clinicians should adjust their workflows to include remote care; payers should start offering the right financial incentives for providers, to stimulate the real adoption of telecare; and the technology infrastructure should be ready to support the services. One reason why clinical workflows do not usually support telehealth is because payers, traditionally, do not reimburse for such services. Luckily, the situation is now changing, as new policies for reimbursement are being applied. As an example, Medicare started adopting a policy of not reimbursing hospitals for preventable hospital readmissions within 30 days of discharge of patients with heart attacks, congestive HF, and pneumonia. Such a new approach also has prompted some providers to invest in telehealth services, but many of them, as well as many vendors, are still looking for the most appropriate use cases, and dealing with reimbursement issues, as they innovate. Analysts observe that telehealth may be helpful for monitoring patients with congestive $\mathrm{HF}$ at home, but a relevant issue to account for is related to the large amount of data that home, and even in-hospital, monitoring devices generate. Both transmission-, storage-, and privacy-related concerns arise when dealing with such a huge amount of expected data, and information technologies systems and clinician habits are usually not set up to process the additional amount of data provided by telehealth monitoring systems. However, the implementation of effective information technologies systems alone is not sufficient for the proper functioning of telehealth services.
New technologies cannot simply be introduced into a health care or homecare environment without providing proper training to staff and patients. Training, technical support, and usability and quality of programs all need to be addressed for telehealth services and infrastructures to be successful. Home telehealth as a method of health care delivery is not an integral and established part of the education and training of health care professionals. As a consequence, targeted professional education and training should precede the deployment of home telehealth, and continuing education programs should be developed. The success of home telehealth relies on the competency of the home telehealth providers, who must have expert assessment skills and must communicate effectively while engaging in equipment-related tasks. Basic computer skills are paramount for correct operation of home telehealth devices. In fact, some of the home telehealth providers' job responsibilities differ to the job responsibilities of traditional home care visits. For example, they may be required to learn how to install, calibrate, and operate the technology and to recognize and solve technical problems. The effects of introducing home telehealth into the health care system remain unclear, because many studies evaluate the clinical outcomes and not the systematic impacts. The transition period may be long because of the training of staff and changes to the system infrastructure, so the benefits of home telehealth may be realized only several years after its implementation. A gradual shift to a home telehealth-supported delivery model is the only realistically advisable type of shift.

Some of the questions to consider before implementation of home telehealth technologies are:

- Which illness/disease conditions exhibit the highest resource use?

- What types of resources are available and required to implement new models for health care provisioning?

- What are the most effective home telehealth technologies to support services provision?

- Does the evidence support using the technology to achieve desired patient outcomes or reduce service delivery costs?

- What factors contribute to the complexity of implementation?

Stakeholders' involvement in the design process is a key factor for the successful deployment of telehealth solutions. ${ }^{5,54}$

Several institutions tried and are still trying to estimate the economic impacts of hospital readmissions; as an example, Fast Adoption of Significant Technologies (FAST) estimates that US \$290 billion of health care expenditures could be 
avoided each year if medication adherence were improved. ${ }^{55}$ Reducing readmissions rates has become a high priority for policymakers and payers seeking to improve health care quality and contain costs. Rehospitalization also appears to increase the risk of health complications, resulting in greater functional and cognitive impairments for patients. Most of the cost-related evaluations of telehealth discussed within this paper agree on confirming the economic benefits generated by even simple approaches: although a sometime considerable initial investment is required, telehealth is able to quickly compensate for this initial expenditure and generate savings.

Analyses of the data provided by the VHA telehealth program from a cohort of 17,025 patients show the benefits of a $25 \%$ reduction in numbers of bed days of care, a $19 \%$ reduction in numbers of hospital admissions, and a mean satisfaction score rating of $86 \%$, after enrollment into the program..$^{21}$ The cost of the VHA telehealth program is around US $\$ 1,600$ per patient per annum, substantially less than other non-institutional care programs and nursing home care. ${ }^{23}$ A very detailed and systematic review of the key indicators for assessing telehomecare cost-effectiveness ${ }^{56}$ identified research reports on telehomecare, published between 1997 and 2007. Among the selected studies, 23 were appropriate for comparison of costs in various ways: $70 \%$ were in the United States, 15 of 23 were randomized control trials, and 48\% were published between 2003 and 2007. Teleconsultation was intended as telemonitoring in the services, and the report showed that the total cost, the cost per patient, and the cost per visit were all reduced by telehomecare. The report also concluded that standardization of cost outcomes should be implemented in order to help funding agencies better understand the importance of telehomecare. The Hospital at Home ${ }^{\circledR}$ model developed by the Johns Hopkins University Schools of Medicine and Public Health to provide acute hospital-level care within patients' homes, can be adapted and implemented with different levels of complexity. A 2012 study ${ }^{57}$ reports that patients enrolled within a telehealth program based on the Hospital at Home ${ }^{\circledR}$ model show comparable or even better clinical outcomes with respect to similar inpatients, but they express higher satisfaction levels. The program achieved 19\% savings over costs for similar inpatients, mainly thanks to a shorter average length of stay, and the use of fewer lab and diagnostic tests, compared with similar patients in hospital acute care, thus ensuring clinical quality, affordability, and positive patient experience.

As a final remark about the cost-related perspective on telehealth for HF management, it is interesting to point out that the Center for Technology and Aging (CTA) and the Partners HealthCare Center for Connected Health $(\mathrm{CCH})$ developed a return on investment evaluation tool ${ }^{58}$ to help health care organizations assess the intrinsic financial costs and benefits of RPM technologies. In particular, RPM for CHF has shown significant cost savings; however, financial factors, including a lack of Medicare reimbursement, have limited the use of RPM in many health care practices. To support the adoption and sustainability of RPM, the tool may generate aggregate findings to identify the benefits of RPM technologies for patients, providers, and family caregivers; to design strategies for program deployment; and to forecast realistic program outcomes. It is a significant, and almost unique, example of a tool designed in a market-oriented approach, and not only in a research-oriented flavor, and it is expected that in the near future several similar tools will appear, to support policymakers and health care providers in assessing their RPM projects and services.

\section{Conclusion}

Moving from the observation that a huge number of research papers is already available in the literature, dealing with the evaluation of telemedicine solutions to support patients affected by HF, and given that their exhaustive analysis would not bring a relevant contribution to the interested community, this short review tried to focus on the practical implications, and realistically expectable outcomes, of RPM solutions based on the technologies current available in the market. Most of the experimental services based on test populations of significant size, and adhering to sound scientific rigor, are quite recent, and basically agree on finding that telehealth management systems may save money, for the patients, the health providers, and the payers, compared to traditional approaches in care provisioning. At the same time, it has been proved that patient adherence and satisfaction may be very high when a suitable service is designed and tailored to the specific user's needs.

\section{Disclosure}

The author reports no conflicts of interest in this work.

\section{References}

1. van den Broek G, Cavallo F, Wehrmann C, editors. AALIANCE Ambient Assisted Living Roadmap. Amsterdam: IOS Press BV; 2010.

2. Romanow RJ. Building on Values: The Future of Health Care in Canada. HCC Final Report. Saskatoon: Commission on the Future of Health Care in Canada; 2002. Available from: http://www.cbc.ca/healthcare/ final_report.pdf. Accessed on January 9, 2014.

3. Bowles KH, Hanlon AL, Glick HA, et al. Clinical effectiveness, access to, and satisfaction with care using a telehomecare substitution intervention: a randomized controlled trial. Int J Telemed Appl. 2011;2011:540138. 
4. Pekmezaris R, Mitzner I, Pecinka KR, et al. The impact of remote patient monitoring (telehealth) upon Medicare beneficiaries with heart failure. Telemed J E Health. 2012;18(2):101-108.

5. Klersy C, De Silvestri A, Gabutti G, et al. Economic impact of remote patient monitoring: an integrated economic model derived from a metaanalysis of randomized controlled trials in heart failure. Eur J Heart Fail. 2011;13(4):450-459.

6. Bhimaraj A. Remote monitoring of heart failure patients. Methodist Debakey Cardiovasc J. 2013;9(1):26-31.

7. Ruggiero C, Sacile R, Giacomini M. Home telecare. JTelemed Telecare. 1999;5(1):11-17.

8. California Telehealth Advancement Act of 2011 (AB 415), Business \& Professions Code section 2290.5. Available from: http://codes.lp.findlaw. com/cacode/BPC/1/d2/5/12/s2290.5. Accessed January 9, 2014.

9. Fisher EB, Boothroyd RI, Coufal MM, et al. Peer support for selfmanagement of diabetes improved outcomes in international settings. Health Aff (Millwood). 2012;31:1130-1139.

10. Jackson KM, Scott KE, Graff Zivin J, et al. Cost-utility analysis of telemedicine and ophthalmoscopy for retinopathy of prematurity management. Arch Ophthalmol. 2008;126(4):493-499.

11. Baker LC, Johnson SJ, Macaulay D, Birnbaum H. Integrated telehealth and care management program for Medicare beneficiaries with chronic disease linked to savings. Health Aff (Millwood). 2011;30(9): 1689-1697.

12. Craig J, Chua R, Russell C, Patterson V, Wootton R. The cost-effectiveness of teleneurology consultations for patients admitted to hospitals without neurologists on site. 1: A retrospective comparison of the case-mix and management at two rural hospitals. J Telemed Telecare. 2000;6 Suppl 1:S46-S49.

13. Dimmick SL, Burgiss SG, Robbins S, Black D, Jarnagin B, Anders M. Outcomes of an integrated telehealth network demonstration project. Telemed J E Health. 2003;9(1):13-23.

14. Leggett PF, Graham L, Steele K, et al. Telerheumatology - diagnostic accuracy and acceptability to patient, specialist, and general practitioner Br J Gen Pract. 2001;51(470):746-748.

15. Host JF, Hasan A. Role of telephone monitoring in patients with chronic heart failure: theory and practical implications. Smart Homecare Technology and TeleHealth. 2014;2:1-12.

16. Antonicelli R, Testarmata P, Spazzafumo L, et al. Impact of telemonitoring at home on the management of elderly patients with congestive heart failure. J Telemed Telecare. 2008;14:300-305.

17. Krumholz HM, Parent EM, Tu N, et al. Readmission after hospitalization for congestive heart failure among Medicare beneficiaries. Arch Intern Med. 1997;157(1):99-104.

18. Huntington MK, Guzman AI, Roemen A, Fieldsend J, Saloum H. Hospital-to-Home: a hospital readmission reduction program for congestive heart failure. S. D. Med. 2013;66(9):370-373.

19. Spinsante S, Antonicelli R, Mazzanti I, Gambi E. Technological approaches to remote monitoring of elderly people in cardiology: a usability perspective. Int J Telemed Appl. 2012;2012:104561.

20. Jaarsma T, Brons M, Kraai I, Luttik ML, Stromberg A. Components of heart failure management in home care; a literature review. Eur $J$ Cardiovasc Nurs. 2013;12(3):230-241.

21. Broderick A. The Veterans Health Administration: Taking Home Telehealth Services to Scale Nationally. Case Studies in Telehealth Adoption. Pub 1657, Vol 4. New York, NY: The Commonwealth Fund; 2013

22. US Department of Veterans Affairs, VHA Office of Telehealth Services. Defining Who We Are - Defining Telehealth. Quarterly Newsletter of VHA Office of Telehealth Services. 2011;10(3):2. Available from: http://www.telehealth.va.gov/newsletter/2011/071511-Newsletter Vol10Iss03.pdf. Accessed on January 9, 2014.

23. Darkins A, Ryan P, Kobb R, et al. Care Coordination/Home Telehealth: the systematic implementation of health informatics, home telehealth, and disease management to support the care of veteran patients with chronic conditions. Telemed J E Health. 2008;14(10):1118-1126.

24. Kehle SM, Greer N, Rutks I, Wilt, TJ. Interventions to Improve Veterans' Access to Care: A Systematic Review of the Literature. Washington, DC: Department of Veterans Affairs (US); 2011.
25. Clark RA, Inglis SC, McAlister FA, Cleland JG, Stewart S Telemonitoring or structured telephone support programmes for patients with chronic heart failure: systematic review and meta-analysis. $B M J$. 2007;334:942.

26. Klersy C, De Silvestri A, Gabutti G, Regoli F, Auricchio A. A metaanalysis of remote monitoring of heart failure patients. $\mathrm{J} \mathrm{Am} \mathrm{Coll}$ Cardiol. 2010;55(19):2185.

27. Dang S, Dimmick S, Kelkar G. Evaluating the evidence base for the use of home telehealth remote monitoring in elderly with heart failure. Telemed J E Health. 2009;15:783-796.

28. Maric B, Kaan A, Ignaszewski A, Lear S. A systematic review of telemonitoring technologies in heart failure. Eur J Heart Fail. 2009;11: 506-517.

29. Domingo M, Lupón J, González B, et al. [Noninvasive remote telemonitoring for ambulatory patients with heart failure: effect on number of hospitalizations, days in hospital, and quality of life. CARME (CAtalan Remote Management Evaluation) Study]. Rev Esp Cardiol. 2011;64(4):277-285. Spanish.

30. Martín-Lesende I, Orruño E, Bilbao A, et al. Impact of telemonitoring home care patients with heart failure or chronic lung disease from primary care on healthcare resource use (the TELBIL study randomised controlled trial). BMC Health Serv Res. 2013;13:118.

31. Koehler F, Winkler S, Schieber M, et al; TIM-HF Investigators. Telemedical Interventional Monitoring in Heart Failure (TIM-HF), a randomized, controlled intervention trial investigating the impact of telemedicine on mortality in ambulatory patients with heart failure: study design. Eur J Heart Fail. 2012;12:1354-1362.

32. Koehler F, Winkler S, Schieber M, et al; Telemedical Interventional Monitoring in Heart Failure Investigators. Impact of remote telemedical management on mortality and hospitalizations in ambulatory patients with chronic heart failure: the telemedical interventional monitoring in heart failure study. Circulation. 2011;123(17):1873-1880.

33. Takahashi PY, Pecina JL, Upatising B, et al. A randomized controlled trial of telemonitoring in older adults with multiple health issues to prevent hospitalizations and emergency department visits. Arch Intern Med. 2012;172(10):773-779.

34. Madigan E, Schmotzer BJ, Struk CJ, et al. Home health care with telemonitoring improves health status for older adults with heart failure. Home Health Care Serv Q. 2013;32(1):57-74.

35. Technologies for Remote Patient Monitoring in Older Adults. Position Paper Discussion Draft. Oakland, CA: Centre for Technology and Aging; 2009.

36. Remote Patient Monitoring Technologies - A Strategic Assessment (Technical Insights) [webpage on the Internet]. Frost and Sullivan Research Service; 2010. Available from: http://www.frost.com/prod/ servlet/report-brochure.pag?id=D21E-01-00-00-00\#report-overview. Accessed on January 9, 2014.

37. Antonicelli R, Ripa C, Abbatecola AM, Capparuccia CA, Ferrara L, Spazzafumo L. Validation of the 3-lead tele-ECG versus the 12-lead tele-ECG and the conventional 12-lead ECG method in older people. J Telemed Telecare. 2012;18:104-108.

38. Antonicelli R, Mazzanti I, Abbatecola AM, Parati G. Impact of home patient telemonitoring on use of $\beta$-blockers in congestive heart failure. Drugs Aging. 2010;27(10):801-805.

39. Ciere Y, Cartwright M, Newman SP. A systematic review of the mediating role of knowledge, self-efficacy, and self-care behaviour in telehealth patients with heart failure. J Telemed Telecare. 2012;18:384-391.

40. Barbosa Neves B, Amaro F. Too old for technology? How the elderly of Lisbon use and perceive ICT. The Journal of Community Informatics. $2012 ; 8(1)$

41. Roupa Z, Nikas M, Gerasimou E, et al. The use of technology by the elderly. Health Science Journal. 2010;4(2):118-126.

42. Spinsante S, Gambi E. Remote health monitoring by OSGi technology and digital TV integration. IEEE Transactions on Consumer Electronics. 2012;58(4):1434-1441.

43. Agha Z, Schapira RM, Laud PW, McNutt G, Roter DL. Patient satisfaction with physician-patient communication during telemedicine. Telemed J E Health. 2009;15(9):830-839. 
44. Mair F, Whitten P. Systematic review of studies of patient satisfaction with telemedicine. BMJ. 2000;320-1517.

45. Gustke SS, Balch DC, West VL, Rogers LO. Patient satisfaction with telemedicine. Telemed J. 2000;6(1):5-13.

46. Jerant AF, Azari R, Martinez C, Nesbitt TS. A randomized trial of telenursing to reduce hospitalization for heart failure: patientcentered outcomes and nursing indicators. Home Health Care Serv $Q$. 2003;22(1):1-20.

47. Gorst SL, Armitage CJ, Brownsell S, Hawley MS. Home telehealth uptake and continued use among heart failure and chronic obstructive pulmonary disease patients: a systematic review. Ann Behav Med. Epub April 25, 2014.

48. Joseph V, West RM, Shickle D, Keen J, Clamp S. Key challenges in the development and implementation of telehealth projects. J Telemed Telecare. 2011;17:71-77.

49. Cimperman M, Makovec Brenčič M, Trkman P, de Leonni Stanonik M. Older Adults' Perceptions of Home Telehealth Services. Telemedicine and e-Health. 2013;19(10):786-790.

50. Moreira Becker Reginatto B. Understanding Barriers to Wider Telehealth Adoption in the Home Environment of Older People: An Exploratory Study in the Irish Context. International Journal on Advances in Life Sciences. 2012;4(3,4):63-76.

51. Litan R. Vital Signs via Broadband: Remote Health Monitoring Transmits Savings, Enhances Lives. October Report. 2008. Available from: https://www.corp.att.com/healthcare/docs/litan.pdf. Accessed January 9, 2014
52. Population Ageing and Development: Ten Years After Madrid. United Nations Department of Economics and Social Affairs; 2012. Available from: http://www.un.org/en/development/desa/population/publications/ pdf/popfacts/popfacts_2012-4.pdf. Accessed February 27, 2014.

53. Hospital Readmissions in Europe. Qualcomm Life, The Burrill Report. Available from: http://www.burrillreport.com/content/ Hospital-Readmissions-Europe_HiRes.pdf. Accessed February 27, 2014.

54. Tran K, Polisena J, Coyle D, et al. Home Telehealth for Chronic Disease Management. [Technology report number 113]. Ottawa: Canadian Agency for Drugs and Technologies in Health; 2008.

55. Detailed Technology Analysis: Home Telehealth. New England Healthcare Institute. Fast Adoption of Significant Technologies (FAST); 2009. http://www.nehi.net/writable/publication_files/file/detailed_technology_analysis_home_telehealth.pdf. Accessed January 9, 2014.

56. Rojas SV, Gagnon MP. A systematic review of the key indicators for assessing telehomecare cost-effectiveness. Telemed $J$ E Health. 2008;14(9):896-904.

57. Cryer L, Shannon SB, Van Amsterdam M, Leff B. Costs for 'hospital at home' patients were 19 percent lower, with equal or better outcomes compared to similar inpatients. Health Aff (Millwood). 2012;31(6): $1237-1243$

58. ROI Calculator for Heart Failure Monitoring [webpage on the Internet]. Center for Technology and Aging, Partners HealthCare Center for Connected Health. Available from: http://www.telemedroi.com/\#home. Accessed February 27, 2014
Smart Homecare Technology and TeleHealth

\section{Publish your work in this journal}

Smart Homecare Technology and TeleHealth is an international, peer-reviewed, open access online journal publishing original research, reviews, editorials and commentaries on the application of technology to support people and patients at home and in assisted living centers to optimize healthcare and management resources. Specific topics in the journal include: Development and application of

\section{Dovepress}

devices within the home and embedded in appliances; Healthcare provider communication and education tools; and drug ordering and adherence. The manuscript management system is completely online and includes a very quick and fair peer-review system, which is all easy to use. Visit http://www.dovepress.com/ testimonials.php to read real quotes from published authors. 